\title{
Regeneration and transient gene expression in protoplasts of Draparnaldia (chlorophytes), an emerging model for comparative analyses with basal streptophytes
}

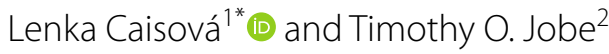

\begin{abstract}
Background: Green plants comprise two lineages: (1) the streptophytes that colonised land and (2) the chlorophytes that have adaptations to land but remained mostly aquatic. To better understand what made streptophytes so successful, we are currently establishing the chlorophyte alga Draparnaldia sp. (Chaetophorales, Chlorophyceae) as a model for comparative analyses between these two lineages. However, establishing Draparnaldia as a valuable model requires that it can be transformed. Thus, our goal is to develop a transformation protocol for this alga.
\end{abstract}

Results: We have established the first transformation protocol for Draparnaldia. This protocol is based on protoplast transformation by electroporation. It includes instructions on protoplast isolation, regeneration and transient transfection. It also provides a list of the effective selective agents for future Draparnaldia transformations.

Conclusions: Our protocol opens a way for Draparnaldia functional genomics analyses. Moreover, it also provides an important base for establishment of stable transformation.

Keywords: Chlorophytes, Colonization of land, Draparnaldia, Land plants, Model organism, Protoplasts, Streptophytes, Transformation

\section{Background}

Colonization of land by plants was a major transition on Earth. Although it is generally accepted that land plants evolved from freshwater streptophyte algae, their key properties enabling such a transition are still poorly understood [1-5 and citations therein]. To examine these properties several basal land plant and streptophyte algal models, such as Anthoceros [6], Chara [7], Closterium [8], Klebsormidium [9], Marchantia [10], Mougeotia [11] and Physcomitrella [12] are (or are currently being) established. However, there are also many chlorophytes (a sister lineage to streptophyte algae and land plants) that moved to terrestrial habitats and morphologically even

\footnotetext{
*Correspondence: Icaisova@gmail.com

${ }^{1}$ Centre for Plant Sciences, Faculty of Biological Sciences, University of Leeds, Woodhouse Lane, Leeds LS2 9JT, UK

Full list of author information is available at the end of the article
}

resemble mosses. This raises the important question of why no land plants have evolved from chlorophytes?

To better understand what made streptophytes so successful, we are currently establishing the freshwater multicellular chlorophyte alga Draparnaldia sp. (Chaetophorales, Chlorophyceae) as a model for comparative analyses between these two lineages. For the phylogenetic position of Draparnaldia in the green tree of life see Fig. 1. Draparnaldia possesses a broad range of adaptations to aquatic and terrestrial habitats. It displays complex morphology similar to mosses and some streptophyte algae: branching filaments, rhizoids with apical growth, and tissue specialization [13-15]. It also reproduces in a similar manner as many streptophyte algae, for Draparnaldia life cycle see Fig. 2. Moreover, it is well positioned phylogenetically. It belongs to the Chaetophorales (fig. 1 in Ref. [13]), whose species range from unbranched filaments with a single-celled 


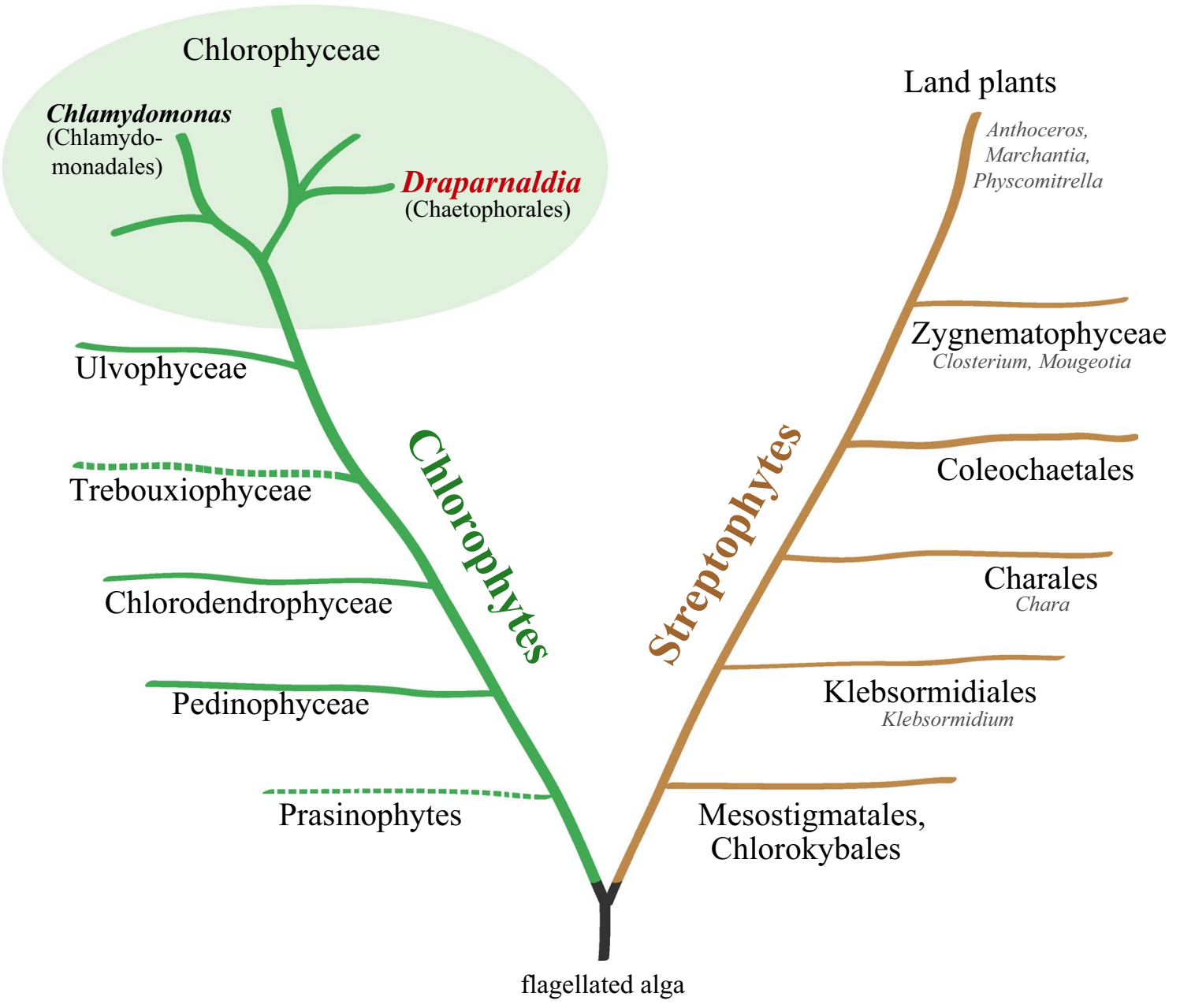

Fig. 1 Phylogenetic position of Draparnaldia in the green tree of life. In addition to Draparnaldia, a well-established Chlamydomonas chlorophyte model species as well as several streptohyte models are shown. The tree schematic is based on Ref. [4, 65-67]. Dash-lined lineages are probably not monophyletic

attachment to branched filaments with multi-celled rhizoids. Thus, it enables comparison of complex filamentous body development between chlorophytes and basal streptophytes. All these features make Draparnaldia an attractive model to distinguish properties that are unique to streptophytes from those that are common to both chlorophytes and streptophytes.

Draparnaldia transcriptome has recently been sequenced and will be published in a separate paper. In addition, there is a plan for genome sequencing. However, establishing Draparnaldia as a valuable model also requires that it is genetically transformable. From the variety of methods for plant and algal transformation [11, 16-21] we focused on transformation via protoplasts. The reasons for this choice were threefold: (1) It permits regeneration of the alga from a single cell, which is crucial for developmental studies. (2) Exogenous DNA can be delivered into the cell using different methods, such as electroporation $[22,23]$ or polyethylene glycol (PEG)-mediated transformation [24, 25]. (3) A few reports about protoplast isolation and regeneration in filamentous chlorophytes exist [26-28], suggesting that protoplast transformation might be possible. Here, we present the first protocol for protoplast transformation of Draparnaldia. The protocol consists of four parts: protoplast isolation, regeneration, transient transfection via electroporation, and identification of effective selective agents for future Draparnaldia transformations.

\section{Methods}

Chemicals and equipment

All chemicals were of highest purity grade and were purchased from Bayer, Duchefa Biochemie, Merck, 


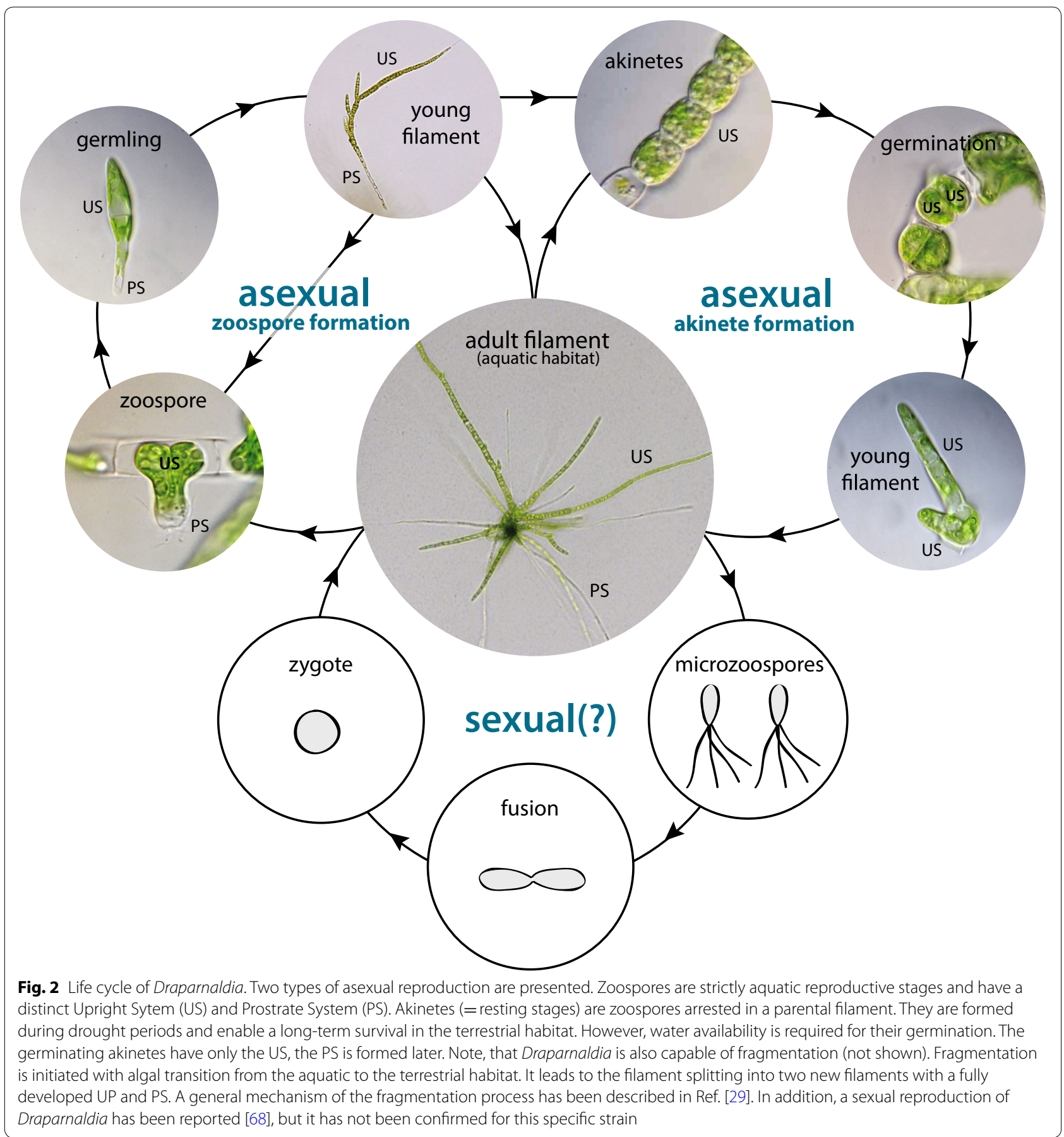

New England BioLabs, Roth, Serva, Sigma-Aldrich or Thermo Fisher Scientific (Additional file 1: Supplement 1a). Equipment list with suppliers is provided in Additional file 1: Supplement 1b.

\section{Reagent setup}

D-Mannitol, $0.5 \mathrm{M}$ was prepared one day before use. $9.1 \mathrm{~g}$ of D-mannitol was dissolved in $100 \mathrm{~mL}$ of $\mathrm{dH}_{2} \mathrm{O}$. The $\mathrm{pH}$ was adjusted to 7.2 with $\mathrm{NaOH}$ and/or $\mathrm{HCl}$. The solution was sterilized with a $0.2-\mu \mathrm{m}$ filter and stored at the room temperature.

Driselase, $2.5 \%$ stock solution was prepared just before use. $0.25 \mathrm{~g}$ driselase was dissolved in $10 \mathrm{~mL}$ of $0.5 \mathrm{M}$ Mannitol solution (in $15 \mathrm{~mL}$ falcon). After that it was vortexed and wrapped with the aluminum foil, incubated on a shaker $(40 \mathrm{rpm})$ for $30 \mathrm{~min}$ at $4{ }^{\circ} \mathrm{C}$. This was followed 
by its centrifugation $(2500 \times g$ for $10 \mathrm{~min})$ and filter sterilization using a $0.2-\mu \mathrm{m}$ filter.

Regeneration medium 10 and 1 (RM10 and RM1) was derived from Growth medium (GM) by adding D-mannitol and calcium chloride (Table 1). Their names refer to the final concentration of calcium chloride. For RM10, $10 \mathrm{~mL}$ of Stock solution 1 was mixed with $196.6 \mu \mathrm{L}$ of Stock solution 2. For RM1, $9 \mathrm{~mL}$ of Stock solution 1 was mixed with $1 \mathrm{~mL}$ of RM10. The media and stock solutions can be stored at $4{ }^{\circ} \mathrm{C}$ for at least 2 weeks.

\section{Draparnaldia origin}

The algal strain used in this study was Draparnaldia sp. CCAC 6921. The strain originates from a dry bank of the river 'Rio Picocca' in Sardinia, Italy. It was isolated from a leaf surface. The strain was identified by sequencing of the nuclear rRNA genes (18S, 5.8S, ITS2 and partial 28S; $2397 \mathrm{bp}$ ) according to Ref. [29]. The resulting sequence was most closely related to Draparnaldia glomerata CCAP 418/2, from which it differed by 2 nucleotides in ITS2 and $28 \mathrm{~S}$ rDNA. The newly determined sequence is available under the accession number LR597279, Project: PRJEB33155 from the European Nucleotide Archive (ENA), [30]. The axenic strain of Draparnaldia sp. CCAC 6921 can be purchased from the Central Collection of Algal Cultures (CCAC; University of Duisburg-Essen).

\section{Draparnaldia cultivation}

Draparnaldia sp. was cultivated axenically using aerated liquid culture. The growth medium (GM) was based on Bold's basal medium [31]. Four vitamins were added, Vitamin $B_{12}(0.6 \mu \mathrm{g} / \mathrm{L}),(+)$-Biotin $(3 \mu \mathrm{g} / \mathrm{L})$, Thiamine $\cdot \mathrm{HCl}$ $(300 \mu \mathrm{g} / \mathrm{L})$, Niacinamide $(0.3 \mu \mathrm{g} / \mathrm{L})$. The $\mathrm{pH}$ was adjusted to 6.6. Both filaments and protoplasts were grown under standard conditions as described previously [13]. A general guide for Draparnaldia cultivation, including the long-term storage and the recipe for GM, is provided in Additional file 2.

\section{Protoplast isolation}

Protoplasts were isolated according to the protocol originally developed for Physcomitrella patens [16], with several modifications. The modified version of the protocol is described in the Results.

\section{Protoplast regeneration}

The protocol for protoplast regeneration in liquid medium was developed in three steps.

1. Regeneration Medium (RM). GM was supplemented with mannitol for osmotic stabilisation of protoplasts and with calcium chloride to promote cell wall regeneration and subsequent division. The optimal concentration of mannitol was determined stepwise. Initially, Draparnaldia filaments were exposed to different concentrations of mannitol $(0.2-0.5 \mathrm{M})$. This revealed that filaments can grow only below $0.35 \mathrm{M}$ mannitol. Therefore, the lowest mannitol concentration capable of maintaining viable protoplasts was determined and chosen as the optimum. For $\mathrm{CaCl}_{2}$, the optimal concentration was determined by resuspending protoplasts in a 6-well culture plate containing $3 \mathrm{~mL} /$ well of RM comprising GM, mannitol and one of six concentrations of $\mathrm{CaCl}_{2}(0.17,1$, $2.5,5,7.5,10 \mathrm{mM}$ ). This range was selected because $0.17 \mathrm{mM}$ is the concentration used in GM of Draparnaldia and $10 \mathrm{mM}$ is one of the highest concentrations used in protoplast regeneration of higher plants [32]. Protoplasts were observed daily. Selection criteria for the optimal calcium chloride concentration included $>85 \%$ survival rate of protoplasts, cell wall formation (visualized using the Calcofluor white staining [29], cell division, filament formation and branching.

2. Optimal plating density for regeneration. Protoplasts were grown at 4 different densities $\left(10^{2}, 10^{3}\right.$, $10^{4}, 10^{5}$ protoplasts $/ \mathrm{mL}$ ) using the RM developed in step (1). These values reflect optimal densities found across many algal and plant species [33-35]. Morphological observations were made 5, 10 and 20 days after protoplast inoculation. The plating density was considered 'optimal' when all surviving protoplasts were able to regenerate into branched filaments.

3. Filament regeneration on GM. RM was beneficial for early phases of regeneration. However, the osmoti-

Table 1 Stock solutions for RM10 and RM1

\begin{tabular}{|c|c|c|c|c|c|}
\hline No. & Component & Stock solution & Stock concentration & $\begin{array}{l}\text { Final concentration } \\
\text { in } \mathrm{RM} 10\end{array}$ & $\begin{array}{l}\text { Final } \\
\text { concentration } \\
\text { in RM1 }\end{array}$ \\
\hline 1 & D-Mannitol in GM & $\begin{array}{l}6 \mathrm{~g} / 100 \mathrm{~mL} \mathrm{GM} \\
\text { Dissolve, adjust pH to } 6.6 \text { with } \\
\mathrm{NaOH} / \mathrm{HCl} \text {, autoclave. }\end{array}$ & $0.33 \mathrm{M}$ & $0.33 \mathrm{M}$ & $0.33 \mathrm{M}$ \\
\hline 2 & $\mathrm{CaCl}_{2} \cdot 2 \mathrm{H}_{2} \mathrm{O}$ & $\begin{array}{l}2.94 \mathrm{~g} / 40 \mathrm{~mL} \mathrm{dH} \mathrm{H}_{2} \mathrm{O} \\
\text { Dissolve and autoclave. }\end{array}$ & $0.5 \mathrm{M}$ & $10 \mathrm{mM}$ & $1 \mathrm{mM}$ \\
\hline
\end{tabular}


cum prevented restoration of the typical Draparnaldia morphology. To enable full regeneration, filaments were grown in RM until they began to branch (5-7 days after protoplast isolation). Then, the replacement of RM by GM was initiated. To this end, two strategies were tested. First, RM was completely replaced by GM. Second, RM was replaced gradually by removing $50 \%$ of the RM and adding the same volume of GM on the day when the first branching occurred. The same procedure was repeated again on the following day.

\section{Protoplast transfection}

To transform Draparnaldia protoplasts, an electroporation protocol developed for the green alga Chlamydomonas reinhardtii was adapted [36]. For a detail protocol of how to electroporate Draparnaldia protoplasts see "Results". In brief, to visualize protein expression, the expression plasmid pChlamy_4 [36] was modified to create the YFP-reporter construct pChlamy_4-eYFP (Fig. 3). This plasmid was selected because Chlamydomonas and Draparnaldia are closely related (see Fig. 1) and thus it was more likely that their regulatory elements will be conserved. Before electroporation the plasmid was linearized by ScaI digestion. The electroporation was performed in 0.4-cm electroporation cuvette with a $2 \mathrm{~mm}$ gap using electrical pulses of 300, 400 or 600 V. Each pulse was about $2 \mathrm{~ms}$. Confocal microscopy of protoplasts was performed 3-10 days after electroporation. Images were collected on a Leica TCSSPE confocal microscope (Leica Microsystems, Exton, PA USA) using a $63 \times$ oil immersion objective. YFP was excited with the blue argon ion laser (488 nm), and emitted light was collected between 546 and $583 \mathrm{~nm}$. Chloroplasts were excited with a $561 \mathrm{~nm}$ laser, and emitted light was collected from 570 to $651 \mathrm{~nm}$. The two channels were collected separately, and later superimposed. Bright field images were collected simultaneously with the fluorescence images using the transmitted light detector. Images were processed using Leica Suite X software (version 3.3.0.16799).

\section{Determination of selective agents}

Ten selective agents (including zeocin-a selective marker in pChlamy_4 plasmid) were tested in Draparnaldia (see "Results"). The initial screening was performed with 4 concentrations $(5,25,50,100 \mu \mathrm{g} / \mathrm{mL})$, representing low and high values typically used for plants and algae [e.g. 25, 37-39]. For those agents that were able to kill Draparnaldia, the minimum inhibitory concentration was determined. Experiments used 15-day old regenerated filaments (original plating density 1200 protoplasts/

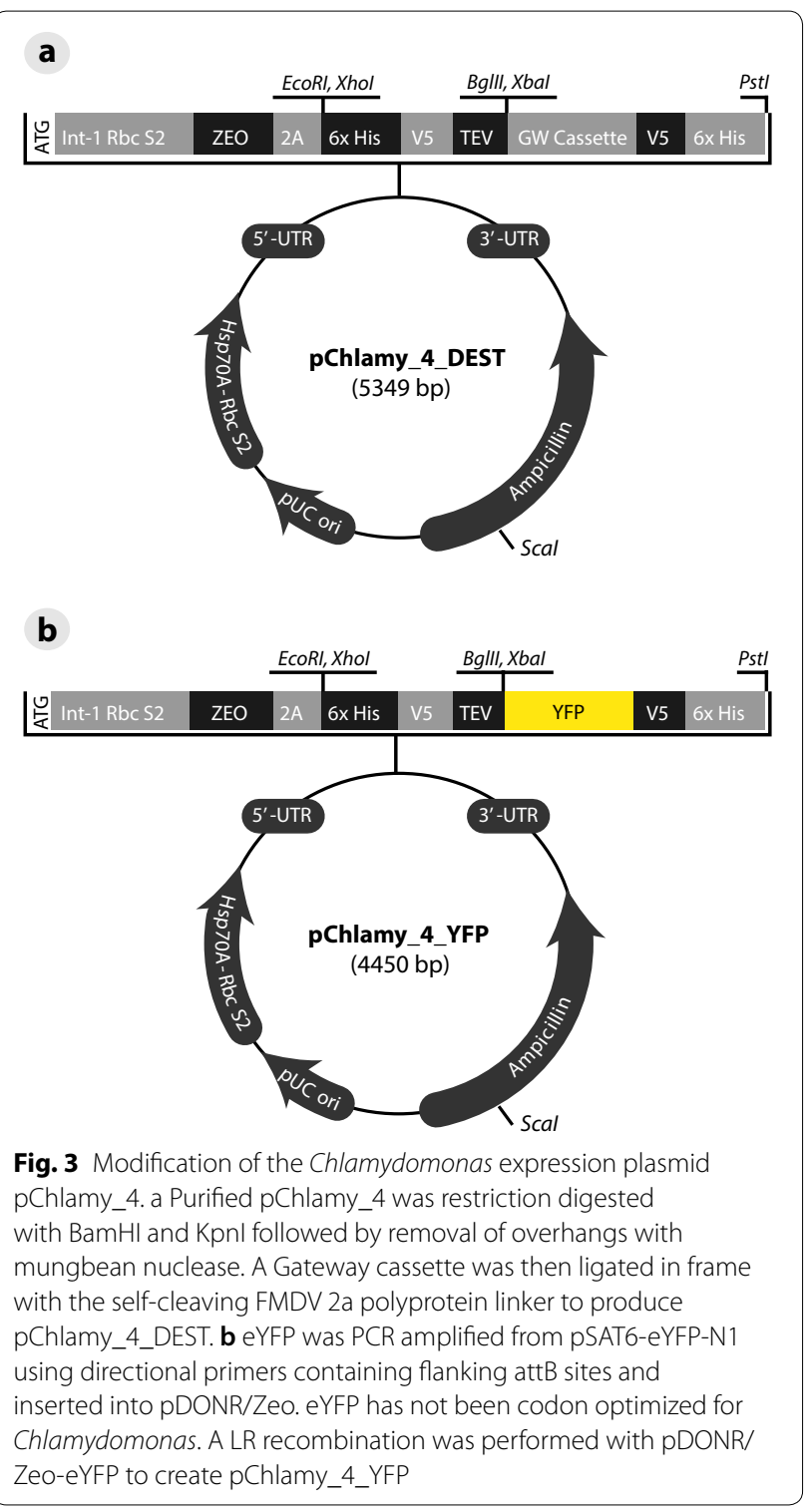

$\mathrm{mL}$ ) growing in liquid GM in 24-well culture plates. One of the wells was a positive control (GM without selective agents). The percentage of surviving (i.e. green) filaments was examined 7 and 14 days after the application of selective agents.

\section{Data collection and evaluation}

All experiments were conducted in three replicates. All observations, except the transformation (see above), were performed directly in the 6- or 24-well culture plates. For establishment of the protoplast regeneration protocol, at least 200 protoplasts or filaments per well were examined. For survival tests of electroporated protoplasts and determination of effective selective agents, all filaments per dish/well were analysed. Standard deviations are shown in each graph and table. 


\section{Results}

\section{Protoplast isolation}

First, we modified the Cove et al. (2009) protocol for protoplast isolation [16]. This included three major changes: (1) We did not grow the alga on agar, because it yielded only $10^{3}$ protoplasts/g of treated biomass. Instead, we used a liquid aerated culture (Fig. 4a), which yielded $10^{6}$ protoplasts/g of treated biomass. (2) We induced cell plasmolysis prior to cell wall digestion (Fig. 4b). This step improved viability of isolated protoplasts from approximately 70-95\%. (3) We added two more purification steps, because the original protocol did not allow to separate protoplasts from cell debris and short filaments. The modified protocol is provided below. It takes $3-4 \mathrm{~h}$ and it yields between $12-16 \times 10^{6}$ protoplasts per a single isolation (Fig. 4c, d).

\section{Protocol for protoplast isolation}

If not otherwise indicated, all steps should be done on a clean bench. All material used for protoplast isolation must be sterile to avoid contamination.

1. Before starting, prepare the biomass, material, media and reagents. For instructions of how to grow the liquid aerated culture of Draparnaldia see Additional file 2.

2. Harvest 3-4 g of fresh biomass of 7-10-day-old aerated culture using the polyester (PET) mesh (pluriSelect) with a pore size of $40 \mu \mathrm{m}$.

Note: 3-4 g of biomass corresponds approximately to $60-90 \mathrm{~mL}$ of culture, depending on the density. $1 \mathrm{~g}$ of fresh biomass yields between $3-4 \times 10^{6}$ protoplasts/mL.
3. Transfer the harvested biomass into a Petri dish $(100 \times 20 \mathrm{~mm})$ using forceps.

4. To introduce plasmolysis, resuspend the biomass in $12 \mathrm{~mL} \mathrm{0.5} \mathrm{M} \mathrm{mannitol} \mathrm{and} \mathrm{seal} \mathrm{the} \mathrm{Petri} \mathrm{dish} \mathrm{with}$ Parafilm.

5. Incubate the Petri dish for 35-40 min on rotator (70 rpm shaking, room temperature).

6. While waiting, prepare $2.5 \%$ driselase solution. For instructions see "Methods" section.

7. Add $4.5 \mathrm{~mL}$ of sterile driselase to the Petri dish with biomass and mannitol. Seal the Petri dish with Parafilm and cover it with aluminium foil to protect protoplasts from strong light.

Note: The final concentration of driselase is $>0.68 \%$.

8. Incubate the Petri dish with the mixture on a rotary shaker (30-40 rpm shaking, room temperature) until the majority of biomass breaks down. This takes about 45-60 min.

Note: Protoplast isolation from older biomass (approximately 15 days old aerated culture) is possible, but not recommended. It takes $2-3 \mathrm{~h}$ longer and it is less efficient. It yields about $10^{3}-10^{4}$ protoplast/g of biomass. The same holds true for non-aerated Draparnaldia cultures.

9. Check the viability of protoplasts under the light microscope. Abundant free-floating protoplasts and remnants of undigested biomass should be present.

Note: An inverted microscope for tissue cultures (e.g. CK $\times 4$, Olympus, Tokyo, Japan) is recommended. It allows to check the status of the protoplasts directly in the Petri dish.

10. To separate protoplasts from the majority of undigested biomass, filter the mixture from the Petri

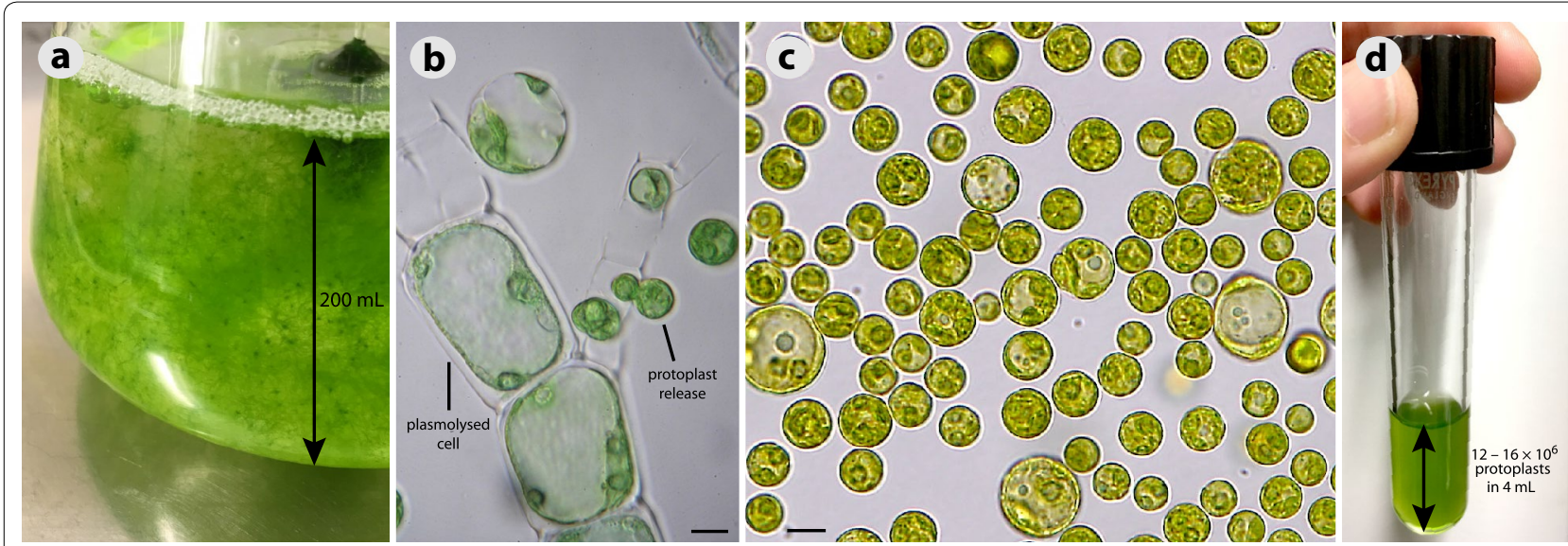

Fig. 4 Draparnaldia protoplasts. a 7-day-old aerated culture, material sufficient for 2-3 isolations. b Cell plasmolysis and protoplast release. c Purified protoplasts. $\mathbf{d}$ Protoplast yield from a single isolation. Scale bar $=10 \mu \mathrm{m}$ 
dish through the polyester (PET) mesh (pluriSelect) with a pore size of $40 \mu \mathrm{m}$.

11. Transfer the filtrate (containing protoplasts and short filaments) back to the Petri dish from Step 10. Seal the Petri dish with Parafilm and cover it with aluminium foil.

12. Incubate the Petri dish containing the filtrate on the rotary shaker for another $15 \mathrm{~min}$ (30-40 rpm shaking, room temperature). This step allows enzymatic digestion of the majority of remaining filament fragments.

13. To separate protoplast from the residual undigested filaments, filter once more through the polyester (PET) mesh (pluriSelect) with a pore size of $15 \mu \mathrm{m}$.

14. Since many protoplasts remain attached to the Petri dish, rinse the Petri dish with an additional $3 \mathrm{~mL} 0.5 \mathrm{M}$ mannitol and filter the suspension through the same mesh as used in Step 13.

15. To separate protoplasts from the remaining cell wall debris, refilter the filtrate from the Step 13 through the polyester (PET) mesh (pluriSelect) with a pore size of $10 \mu \mathrm{m}$.

16. To wash away the rest of driselase, split the filtered protoplast suspension into two $15 \mathrm{~mL}$ glass Screw Cap Culture Tubes.

17. Centrifuge at $50 \times \mathrm{g}$ for $10 \mathrm{~min}$ with the acceleration and brake set to 3 (Eppendorf Centrifuge 5810R, rotor A-4-62).

18. Discard the supernatant.

19. Resuspend the protoplasts in $5 \mathrm{~mL} 0.5 \mathrm{M}$ mannitol by gently rotating the tubes.

20. Repeat Steps 17 and 18.

21. Resuspend the protoplasts in $5 \mathrm{~mL} 0.5 \mathrm{M}$ mannitol by gently rotating the tubes and combine the contents of both tubes.

22. Repeat Steps 17 and 18.

23. Resuspend the protoplasts in $4 \mathrm{~mL} \mathrm{RM} 10$ and set aside a small aliquot (about $400 \mu \mathrm{L}$ ) of the protoplast suspension for quantitative and qualitative assessment of protoplasts.

24. Check the viability of protoplasts using the light microscope (alternatively, use the fluorescein diacetate dye (FDA [40]). Determine protoplast density using a hemocytometer [41] or measure the $\mathrm{OD}_{750}$. Confirm the loss of cell wall using Calcofluor white M2R [42].

Note: In total 15 protoplast isolations have been performed based on this protocol. The viability of protoplast was regularly about $95 \%$. Protoplast density varied from $3-4 \times 10^{6}$ protoplasts $/ \mathrm{mL}$. Protoplasts showed no calcofluor white fluorescence.

\section{Protoplast regeneration}

Next, we established an efficient protocol for regeneration of the isolated protoplasts, i.e. cell wall regeneration and restoration of the original morphology. This was done in three steps.

First, we developed a regeneration medium (RM) that consisted of GM, mannitol and $\mathrm{CaCl}_{2}$. For mannitol, we used a concentration of $0.33 \mathrm{M}$, which was the lowest concentration with nearly $100 \%$ survival rate of protoplasts (Fig. 5). For $\mathrm{CaCl}_{2}, 10 \mathrm{mM}$ was needed for $>85 \%$ protoplast survival rate, but lower concentrations were required for cell division, filament formation and branching (Table 2). Thus, to regenerate the majority of protoplasts into branched filaments, we followed a two-step procedure. Protoplasts were incubated in RM with $10 \mathrm{mM} \mathrm{CaCl}_{2}$ (RM10 medium) for $24 \mathrm{~h}$ and then transferred into RM with $1 \mathrm{mM} \mathrm{CaCl}_{2}$ (RM1 medium).

Second, we determined the optimal protoplast plating density for regeneration. All tested densities $\left(10^{2}-10^{5}\right.$ protoplasts $\left./ \mathrm{mL}\right)$ enabled regeneration into filaments (arrows in Fig. 6a-c). But, only the lowest densities $\left(10^{2}-10^{3}\right.$ protoplasts $\left./ \mathrm{mL}\right)$ allowed for branching (Fig. 6a) and were taken as the optimal plating densities for regeneration.

Third, we replaced RM by GM. Because the complete replacement of RM was too drastic $(>50 \%$ filaments died), gradual replacement (with 100\% survival of filaments) was chosen for the protocol (see Steps 4 and 5).

Two modes of protoplast regeneration were observed: protoplasts either differentiated into zoospores $(=$ motile reproductive bodies) and regenerated (Fig. $7 \mathrm{a}-\mathrm{j}$ ) or they regenerated directly (Fig. 7k-r). The resulting protocol allowed for a successful regeneration of $>90 \%$ protoplasts within 15 (via zoospores) or 20 days (direct regeneration). In both cases, the regenerated filaments resembled the original Draparnaldia morphology and were able to reproduce (ZF in Fig. 7j, r).

\section{Protocol for protoplast regeneration}

1. Pre-incubate protoplasts for $24 \mathrm{~h}$ in $4 \mathrm{~mL}$ RM10 in a glass Screw Cap Culture Tube. Gently resuspend the protoplasts at least $3 \times /$ day.

Note: Do not pre-incubate the protoplasts in Petri dishes or plates. Unlike protoplasts from embryophytes, those of Draparnaldia attach to the surface and cannot be removed without damage. Protoplasts can be kept in RM10 up to 5 days, after that the regeneration rate decreases to about $70 \%$.

2. Adjust the density of protoplasts in RM1 medium to $10^{2}-10^{3} / \mathrm{mL}$. 


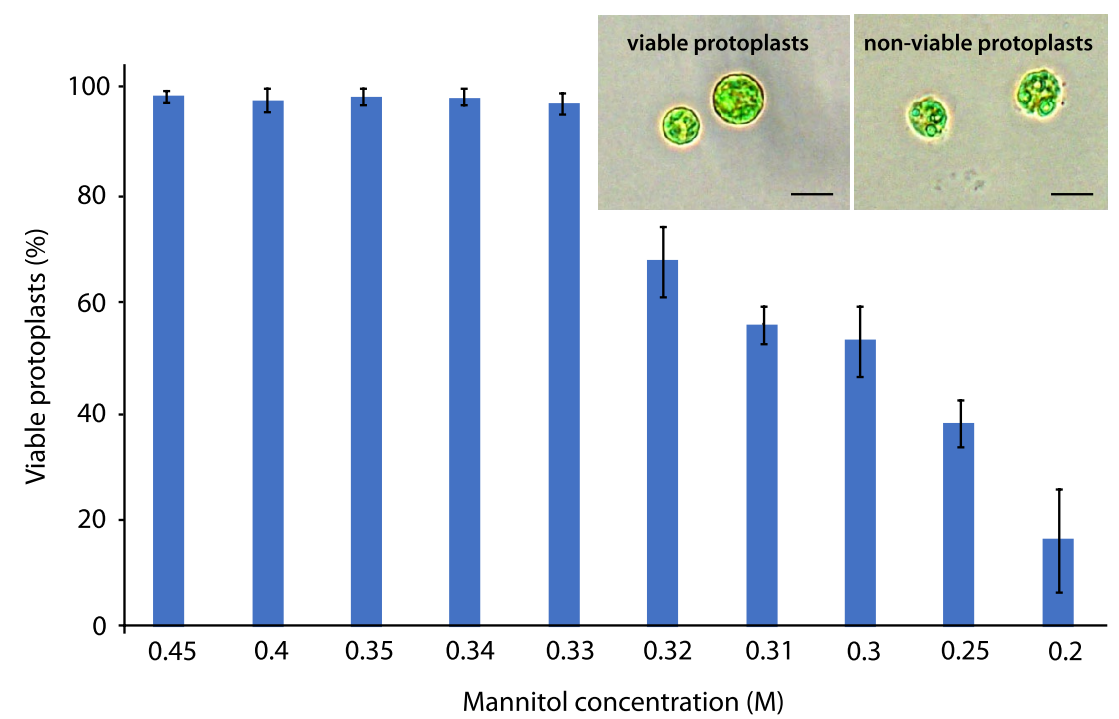

Fig. 5 Effect of mannitol concentration on the viability of protoplasts. Bars represent \pm SD from the mean of 3 replicates consisting of 1000 protoplasts per replicate. The graph is accompanied with light microphotographs showing the viable and non-viable protoplasts. Scale bar $=20 \mu \mathrm{m}$

Table 2 Influence of calcium chloride on protoplast regeneration in Draparnaldia

Calcium chloride Concentrations (mM)

\begin{tabular}{lcccccc} 
& 0.17 & $\mathbf{1}$ & 2.5 & 5 & 7.5 & $\mathbf{1 0}$ \\
\hline Survival rate (\%) & $30.3 \pm 2.5$ & $66.3 \pm 2.5$ & $76.7 \pm 1.5$ & $83.7 \pm 4.7$ & $82.7 \pm 1.5$ & $\mathbf{9 4 . 3} \pm \mathbf{1 . 2}$ \\
Cell wall formation (\%) & $91.7 \pm 1.5$ & $\mathbf{9 9} \pm \mathbf{1 . 7}$ & $99.7 \pm 0.6$ & $98.7 \pm 2.3$ & $99 \pm 1.7$ & $\mathbf{9 9} \pm \mathbf{1}$ \\
Cell division (\%) & $99 \pm 1$ & $\mathbf{9 8 . 3} \pm \mathbf{0 . 6}$ & $94.3 \pm 1.5$ & $91.3 \pm 1.5$ & $87 \pm 3$ & $79.3 \pm 3.5$ \\
Filament formation (\%) & $100 \pm 0$ & $\mathbf{9 8} \pm \mathbf{1}$ & $85 \pm 2$ & $71 \pm 1$ & $67.3 \pm 2.1$ & $49.3 \pm 2.1$ \\
Branching (\%) & $100 \pm 0$ & $\mathbf{9 1 . 7 \pm \mathbf { 2 . 9 }}$ & $73.7 \pm 2.3$ & $49.7 \pm 2.5$ & $29 \pm 1$ & $7.7 \pm 2.5$
\end{tabular}

Concentrations used for protoplast regeneration are in bold and orange background. Values are reported as the mean \pm SD for 3 replicates consisting of 500 protoplasts or filaments per replicate

Note: Use cut filter tips for manipulation with protoplasts. This will eliminate their damage.

3. Incubate protoplasts in RM1 until the first branches occur. Importantly, protoplasts will not regenerate in a glass tube, they must be incubated in a Petri dish or in a culture plate.

Note: Dish (plate) should not be filled to more than $50 \%$ to allow for proper gas exchange. Seal the dish (plate) with Parafilm to avoid contamination and evaporation.

4. As soon as branching is observed (usually 5-7 days after incubation in RM1) remove $50 \%$ of the RM and add the same volume of GM.
Note: The regenerated filaments are firmly attached to the surface using their Prostrate System (PS and dashed line in Fig. 7). Therefore, the majority of them will not be washed out during the media exchange.

5. Repeat Step 4 on the next day.

\section{Transfection of protoplasts}

Next we examined whether protoplasts can be transfected. The transfection was performed using a Hsp70A-Rbc S2::Zeo::FMDV2A::YFP construct (pChlamy_4-eYFP, see Fig. 3) by electroporation at three different voltages. The reporter gene expression 


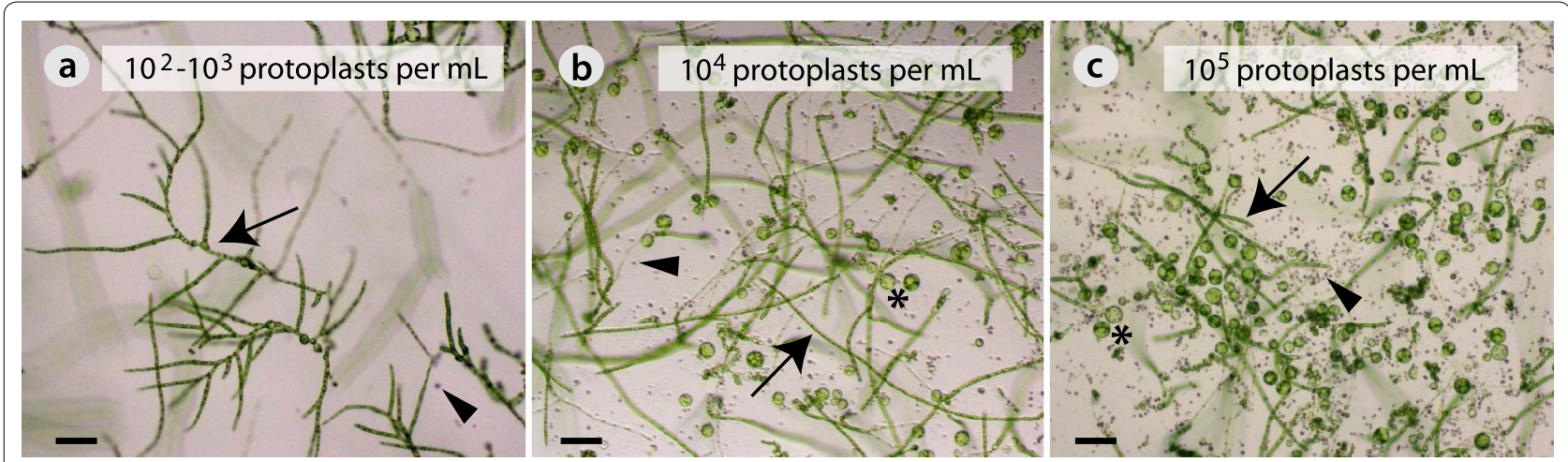

Fig. 6 Influence of protoplast plating density on regeneration. a $10^{2}-10^{3}$ protoplasts $/ \mathrm{mL}$. b $10^{4}$ protoplasts $/ \mathrm{mL}$. c $10^{5}$ protoplasts $/ \mathrm{mL}$. Three stages are shown: filaments (arrow), dead protoplasts (arrow head) and enlarged protoplasts (asterisks). Pictures were taken 20 days after plating. Scale $\mathrm{bar}=80 \mu \mathrm{m}$

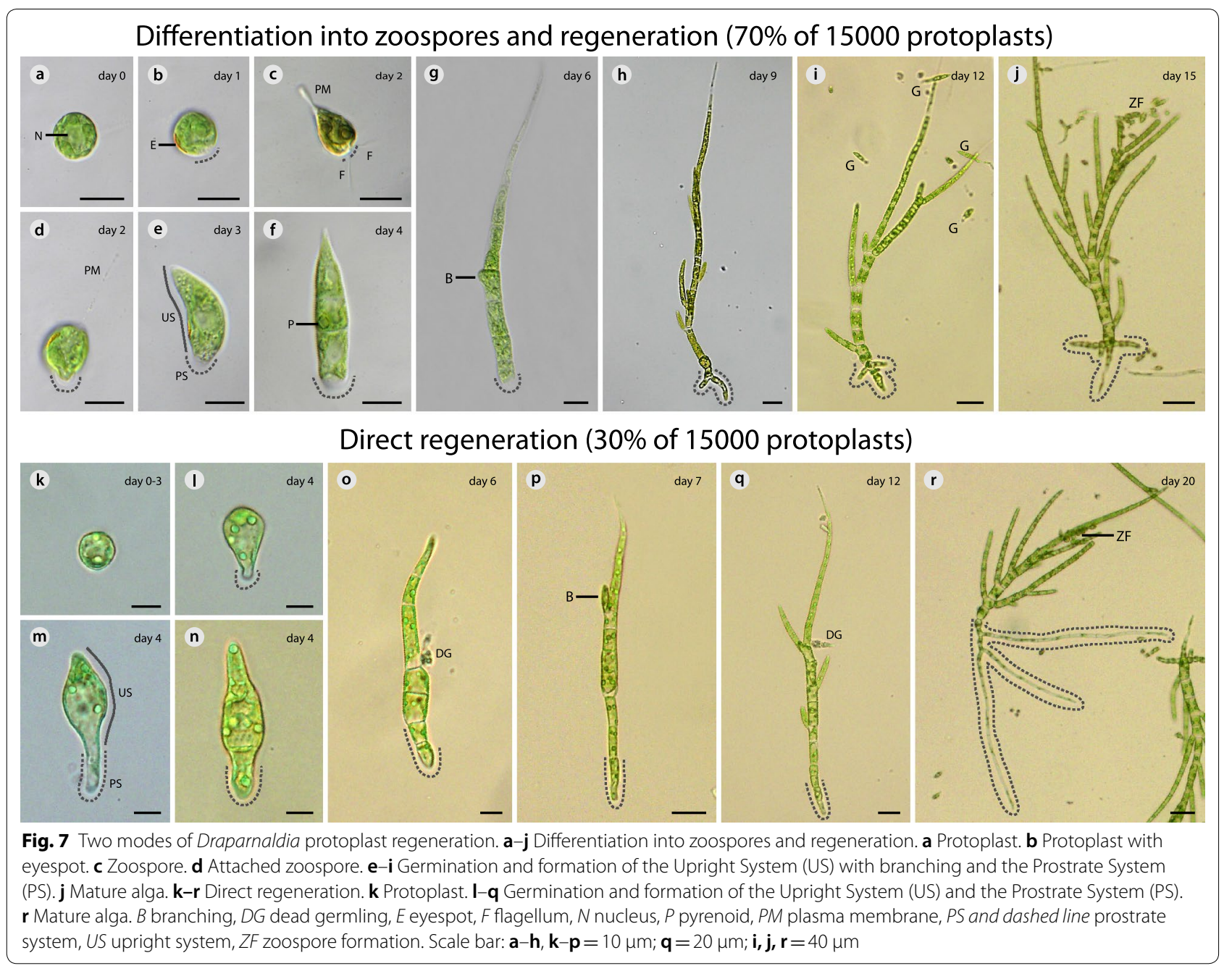

was observed in the majority of living protoplasts (>80\%) electroporated at $300 \mathrm{~V}$ (Fig. $8 \mathrm{a}-\mathrm{c}$ ), but not at 400 and $600 \mathrm{~V}$. Also a negative control did not show any expression, only a weak background fluorescent from the chloroplast was visible (Fig. 8d-f). The expression was transient as it disappeared about 8 days after 
transfection. The survival percentage of electroporated protoplasts depended on the voltage. About $83 \%$ of protoplasts survived $300 \mathrm{~V}, 70 \%$ survived $400 \mathrm{~V}$, and only $50 \%$ survived $600 \mathrm{~V}$. All surviving protoplasts could be fully regenerated (Fig. 8g).

\section{Protocol for transfection of protoplasts}

1. Pre-incubate protoplasts for $24 \mathrm{~h}$ in $4 \mathrm{~mL}$ RM10 in a glass Screw Cap Culture Tube. Gently resuspend the protoplasts at least $3 \times /$ day.

2. Measure protoplast density by $\mathrm{OD}_{750}$ : Pipette $900 \mu \mathrm{L}$ RM10 into the $1.5 \mathrm{~mL}$ semi-micro cuvette

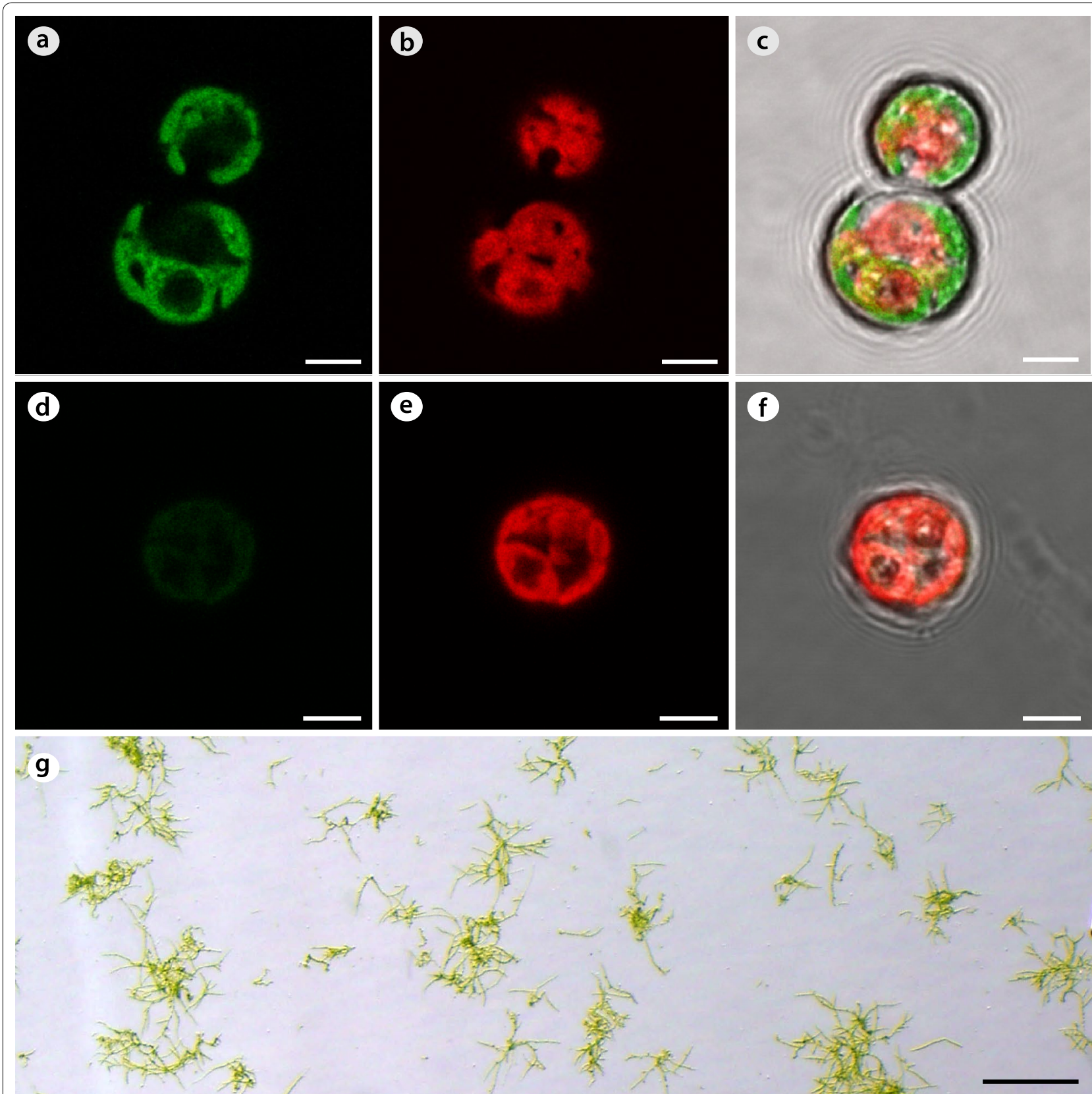

Fig. 8 Expression and regeneration of electroporated protoplasts. a-c Expression in protoplasts. a YFP channel (488 nm). b Chlorophyll channel $(561 \mathrm{~nm})$. c Merged. Pictures were taken 3 days after electroporation. $\mathbf{d}-\mathbf{f}$ Negative control. d YFP channel (488 nm). A weak background fluorescent from the chloroplast is visible. e Chlorophyll channel $(561 \mathrm{~nm})$. $\mathbf{f}$ Merged. Pictures were taken 3 days after electroporation. $\mathbf{g}$ Regenerated filaments from electroporated protoplasts. A picture taken 15 days after electroporation at $300 \mathrm{~V}$. Scale bar: $\mathbf{a}-\mathbf{f}=5 \mu \mathrm{m} ; \mathbf{g}=500 \mu \mathrm{m}$ 
$(12.5 \times 12.5 \times 4.5 \mathrm{~mm})$. Add $100 \mu \mathrm{L}$ of the protoplast suspension and stir gently with a cut filter tip. Perform measurement and throw the sample away. Note: Using cut filter tips for manipulation with protoplasts will eliminate their damage.

3. Resuspend protoplasts from Step 1 in RM10 to a final $\mathrm{OD}_{750}$ of 0.5 .

Note: Again, use cut filter tips for manipulation with protoplasts.

4. Incubate protoplasts on ice for $20 \mathrm{~min}$.

5. Pipette $400 \mu \mathrm{L}$ of the cooled protoplast suspension with cut filter tip into the $1.5 \mathrm{~mL}$ pre-chilled microtube.

6. Add approximately $1 \mu \mathrm{g}$ of linearized plasmid and stir gently with a cut filter tip.

7. Using a cut filter tip, transfer the mixture to a prechilled 0.4-cm electroporation cuvette with a $2 \mathrm{~mm}$ gap.

8. Perform electroporation (electrical pulse $300 \mathrm{~V}$, $2 \mathrm{~ms}$ ).

9. After electroporation, incubate protoplasts on ice for additional $3 \mathrm{~min}$. While waiting, prepare a Petri dish for protoplast regeneration: Place three sterile square coverslips on the bottom of a $60 \times 15 \mathrm{~mm}$ Petri dish and add $7 \mathrm{~mL}$ of RM1.

10. Using a cut filter tip, pipette the protoplast suspension out of the cuvette and placed it on the top of coverslips.

Note: Note that if coverslips are not applied protoplasts attach to the surface of the Petri dish and cannot be collected for microscopy without damage.

11. Seal the Petri dish with Parafilm to prevent evaporation during regeneration.

12. Regenerate protoplasts under standard conditions as described in Ref. [13].

Note: Incubation of protoplasts in the dark is not required.

\section{Selective agents}

Finally, to see if the zeocin resistance in the pChlamy_4 plasmid might be suitable for selection, we evaluated the sensitivity of regenerated protoplasts (the wild-type) to zeocin. In addition, we also tested several other antibiotics and herbicides commonly used for plant and algal selection. Seven out of ten agents tested (including zeocin) were able to kill Draparnaldia. Herbicides were effective within 1 week and antibiotics within 2 weeks. Three antibiotics were ineffective, having no effect on growth. An overview of tested antibiotics and herbicides and the minimum inhibitory concentrations of the effective ones is provided in Table 3 .

\section{Discussion}

In this study we developed a protoplast transformation protocol for Draparnaldia.

The yield of protoplasts from a single isolation $\left(10^{6}\right.$ protoplasts) was comparable with protoplast yields reported for well-established model organisms, such as Physcomitrella patens [16] or Arabidopsis thaliana [43, 44]. Similarly to those organisms, a high protoplast yield could only be achieved when young plant/algal material was used (see "Results"). Probably older plant/algal cells have more complex rigid cell walls, which are more resistant to enzymatic digestion [45-47]. To generate young homogenous biomass, we recommend to grow Draparnaldia in aerated liquid culture (Additional file 2). The combination of fresh medium and aeration transfers the majority of cells (more specifically their protoplasts) into zoospores that give rise to new filaments. The intensity of aeration should be adjusted to ensure homogeneous culture suspension, which is important for equal growth of filaments. Note, that Draparnaldia can also theoretically be transformed by using zoospores (flagellates lacking a cell wall), like gametes of seaweed Ulva [19]. However, Draparnaldia zoospores do not have a strong phototaxis and stay motile for only a few minutes before attaching to the substrate. This makes their collection and purification very difficult and thus prevents them from being used as an efficient source for transformation.

Protoplast regeneration was based on the same parameters as regeneration in other plant/algal organisms $[16,19,46,48-54]$. These parameters were: optimized osmoticum, calcium chloride and plating density of protoplasts (see "Methods" section). Note, that similar to mosses, no addition of plant hormones was needed. Importantly, Draparnaldia protoplasts can be regenerated in Petri dishes or multiwell culture plates, but not in glass tubes. This problem does not seem to be related to the glass material itself (protoplasts can regenerate on coverslips, see "Results"), but rather to some other conditions such as suboptimal light or gas exchange. Another interesting observation was that the majority of protoplasts differentiated into zoospores prior to their regeneration (see "Results"). In fact, this behaviour mimics natural development of Draparnaldia (compare Figs. 2 and 7) and it can be explained by using young biomass for protoplast isolation. Young biomass is known to be favorable for zoospore formation [13, 55]. Moreover, Draparnaldia zoospores can be easily induced by abiotic stresses, such as medium exchange $[13,56]$. Therefore, it is likely that the observed zoospores were either initiated during protoplast isolation (probably when GM was replaced by mannitol) or when protoplasts were transferred into RM. 
Table 3 Effects of different selective agents on Draparnaldia (the wild-type)

Percentage of survival (\%)

\begin{tabular}{|c|c|c|c|c|c|c|c|c|}
\hline \multirow[b]{2}{*}{ Concentrations $(\mu \mathrm{g} / \mathrm{mL})$} & & & & & & & & \\
\hline & $\mathbf{0}$ & 5 & 10 & 25 & 30 & 35 & 50 & 70 \\
\hline Basta & 100 & 100 & 100 & 100 & 90 & 0 & 0 & 0 \\
\hline Chloramphenicol & 100 & 100 & 100 & 100 & 100 & 100 & 100 & 100 \\
\hline Gentamicin & 100 & 100 & 100 & 80 & $\mathbf{0}$ & 0 & 0 & 0 \\
\hline G418 & 100 & 85 & $\mathbf{0}$ & 0 & 0 & 0 & 0 & 0 \\
\hline Hygromycin & 100 & $\mathbf{0}$ & 0 & 0 & 0 & 0 & 0 & 0 \\
\hline Kanamycin & 100 & 100 & 100 & 100 & 100 & 100 & 100 & 100 \\
\hline Paromomycin & 100 & $\mathbf{0}$ & 0 & 0 & 0 & 0 & 0 & 0 \\
\hline Roundup & 100 & 100 & 100 & 100 & 100 & 100 & 83 & 0 \\
\hline Spectinomycin & 100 & 100 & 100 & 100 & 100 & 100 & 100 & 100 \\
\hline Zeocin & 100 & 0 & 0 & 0 & 0 & 0 & 0 & 0 \\
\hline
\end{tabular}

The effective antibiotics and herbicides are in bold and grey background. Note that dead filaments were recognized by pale color. Percentage survival of Draparnaldia filaments based on 3 replicates

We also showed that Draparnaldia protoplasts are capable of being transiently transfected by electroporation. Thus, the next goal will be to determine whether they can be stably transformed. Success in achieving this goal will require optimization of the electroporation as well as testing alternative approaches of transgene delivery. This includes alternative procedures for direct uptake of DNA by protoplasts [19, 25], particle bombardment $[57,58]$ as well as Agrobacterium-mediated conjugative transformation [59]. Also, it will be important to determine whether stable transformation occurs via homologous recombination (like in Physcomitrella, $[60,61]$ ) or random integration (like in most land plants, [62]).

Finally, we demonstrated that regenerated protoplasts (=the wild type) are sensitive to several commonly used selective agents (see "Results". This sensitivity to multiple agents is advantageous for at least two reasons. (1) It might allow a number of molecular applications, including gene stacking, generation of T-DNA mutant libraries and subsequent mutant complementation, and assays where multiple reporter genes are required such as BiFC $[63,64]$. (2) It also suggests that many available antibiotic/herbicide reporter genes could potentially be used for Draparnaldia transformation.

\section{Conclusions}

Here we present the first protoplast transformation protocol for Draparnaldia sp.-the emerging chlorophyte model for comparative analysis with early streptophytes. The protocol yields $10^{6}$ protoplasts per isolation. It allows full regeneration of $>90 \%$ of protoplasts and enables protoplast transient transfection by electroporation. To further expand this toolset, we also identified selective agents that are suitable for selection of future Draparnaldia transformants. This protocol opens a way for functional analyses in Draparnaldia. It also provides the first step towards establishing stable transformation in Draparnaldia. Although our protocol has been established for Draparnaldia, we believe that it can be used as a reference to develop transformation protocols in other chlorophyte as well as streptophyte filamentous algae.

\section{Additional files}

Additional file 1. List of chemicals (Supplement 1a) and equipment (Supplement 1b).

Additional file 2. Cultivation of Draparnaldia. This file contains information on Growth Medium (GM), establishment of actively growing culture, scaling up biomass and establishment of long-term cultures. It also includes instructions about how to assemble and inoculate flasks for aerated liquid culture. Finally, it provides advices how to keep the culture axenic. 


\section{Acknowledgements}

We would like to acknowledge O. Artz (Cologne University, Germany), A. C. Cuming (Leeds University, UK), H. Buschmann (Osnabrück University, Germany) and P. F. Perroud (Marburg University, Germany) for sharing their experience with moss and streptophyte algal transformation. We are grateful to A. C. Cuming and S. Kopriva (Cologne University, Germany) for comments on the manuscript.

\section{Authors' contributions}

LC conceived and designed the study, performed the experiments, wrote the paper. TOJ guided protoplast transfection experiments, performed vector modification, edited the paper. Both authors read and approved the final manuscript.

\section{Funding}

This work was supported by the German Research Foundation (DFG Grant CA 1321/3-1, to LC).

\section{Availability of data and materials}

All data generated or analysed during this study are included in this published article and its additional files.

\section{Ethics approval and consent to participate}

Not applicable.

\section{Consent for publication}

Not applicable.

\section{Competing interests}

The authors declare that they have no competing interests.

\section{Author details}

${ }^{1}$ Centre for Plant Sciences, Faculty of Biological Sciences, University of Leeds, Woodhouse Lane, Leeds LS2 9JT, UK. ${ }^{2}$ Botanical Institute, Cluster of Excellence on Plant Sciences (CEPLAS), University of Cologne, Zülpicher Str. 47b, 50674 Cologne, Germany.

Received: 3 May 2019 Accepted: 6 July 2019

Published online: 12 July 2019

\section{References}

1. de Vries J, Archibald JM. Plant evolution: landmarks on the path to terrestrial life. New Phytol. 2018;217:1428-34.

2. Delwiche $\mathrm{CF}$, Cooper ED. The evolutionary origin of a terrestrial flora. Curr Biol. 2015;25:PR899-R910.

3. Holzinger A, Pichrtová M. Abiotic stress tolerance of charophyte green algae: new challenges for omics techniques. Front Plant Sci. 2016;7:678.

4. Wickett NJ, Mirarab S, Nguyen N, Warnow T, Carpenter E, Matasci N, et al. Phylotranscriptomic analysis of the origin and early diversification of land plants. Proc Natl Acad Sci USA. 2014;111:E4859-68.

5. Wodniok S, Brinkmann H, Glöckner G, Heidel AJ, Philippe H, Melkonian $\mathrm{M}$, et al. Origin of land plants: do conjugating green algae hold the key? BMC Evol Biol. 2011;11:104.

6. Szövényi P, Frangedakis E, Ricca M, Quandt D, Wicke S, Langdale JA. Establishment of Anthoceros agrestis as a model species for studying the biology of hornworts. BMC Plant Biol. 2015;15:98.

7. Nishiyama T, Sakayama H, de Vries J, Buschmann H, Saint-Marcoux D, UIIrich KK, et al. The Chara genome: secondary complexity and implications for plant terrestrialization. Cell. 2018;174(448-64):e24.

8. Tsuchikane Y, Sekimoto H. The genus Closterium, a new model organism to study sexual reproduction in streptophytes. New Phytol. 2019;221:99-104.

9. Hori K, Maruyama F, Fujisawa T, Togashi T, Yamamoto N, Seo M, et al. Klebsormidium flaccidum genome reveals primary factors for plant terrestrial adaptation. Nat Commun. 2014;5:3978.

10. Ishizaki K, Nishihama R, Yamato KT, Kohchi T. Molecular genetic tools and techniques for Marchantia polymorpha research. Plant Cell Physiol. 2016;57:262-70.
11. Regensdorff M, Deckena M, Stein M, Borchers A, Scherer G, Lammers M, et al. Transient genetic transformation of Mougeotia scalaris (Zygnematophyceae) mediated by the endogenous a-tubulin 1 promoter. J Phycol. 2018:54:840-9.

12. Cove DJ, Perroud PF, Charron AJ, McDaniel SF, Khandelwal A, Quatrano RS. The moss Physcomitrella patens: a novel model system for plant development and genomic studies. CSH Protoc. 2009. https://doi.org/10.1101/ pdb.emo115.

13. Caisová L, Melkonian M. The Chaetophorales (Chlorophyceae): a taxonomic revision at family level. Eur J Phycol. 2018;53:381-92.

14. Fritsch FE. The algal ancestry of the higher plants. New Phytol. 1916;15:233-50.

15. Hicks JB. On the similarity between the genus Draparnaldia and the confervoid filaments of mosses. Trans Linn Soc Lond. 1870;27:153-4.

16. Cove DJ, Perroud PF, Charron AJ, McDaniel SF, Khandelwal A, Quatrano RS. Isolation and regeneration of protoplasts of the moss Physcomitrella patens. CSH Protoc. 2009. https://doi.org/10.1101/pdb.prot5140.

17. Hidaka T, Omura M. Transformation of citrus protoplasts by electroporation. J Japan Soc Hort Sci. 1993;62:371-6.

18. Muthukumar B, Joyce BL, Elless MP, Stewart CN Jr. Stable transformation of ferns using spores as targets: Pteris vittata and Ceratopteris thalictroides. Plant Physiol. 2013;163:648-58.

19. Oertel W, Wichard T, Weissgerber A. Transformation of Ulva mutabilis (Chlorophyta) by vector plasmids integrating into the genome. J Phycol. 2015;51:963-79.

20. Oikawa A, Matsuda F, Kikuyama M, Mimura T, Saito K. Metabolomics of a single vacuole reveals metabolic dynamism in an alga Chara australis. Plant Physiol. 2011;157:544-51.

21. Zhang X, Henriques R, Lin SS, Niu QW, Chua NH. Agrobacterium-mediated transformation of Arabidopsis thaliana using the floral dip method. Nat Protoc. 2006;1:641-6.

22. Christou P, Murphy JE, Swain WF. Stable transformation of soybean by electroporation and root formation from transformed callus. Proc Natl Acad Sci USA. 1987;84:3962-6.

23. Niedz RP, McKendree WL, Shatters RJ. Electroporation of embryogenic protoplasts of sweet orange (Citrus sinensis (L.) Osbeck) and regeneration of transgenic plants. Vitro Cell Dev Biol Plant. 2003;39:586-94.

24. Golds T, Maliga P, Koop H-U. Stable plastid transformation in PEG-treated protoplasts of Nicotiana tabacum. Biotechnology. 1993;11:95-7.

25. Schaefer D, Zryd J-P, Knight CD, Cove DJ. Stable transformation of the moss Physcomitrella patens. Mol Gen Genet. 1991;226:418-24.

26. Gabriel M. Formation, growth, and regeneration of protoplasts of the green alga, Uronema gigas. Protoplasma. 1970;70:135-8.

27. Larpent-Gourgaud M, Aumaitre MP. Production et regeneration de protoplasts chez Draparnaldia mutabilis (Chaetophorales, Chlorophyta). Cryptogam Algol. 1987;8:101-6.

28. Marchant HJ, Fowke LC. Preparation, culture, and regeneration of protoplasts from filamentous green algae. Can J Bot. 1977;55:3080-6.

29. Caisová L, Pérez Reyes C, Cruz Álamo V, Martel Quintana A, Surek B, Melkonian M. Barrancaceae: a new green algal lineage with structural and behavioral adaptations to a fluctuating environment. Am J Bot. 2015;102:1482-92.

30. The European Nucleotide Archive. http://www.ebi.ac.uk/ena/data/view/ PRJEB33155. Accessed 22 Jun 2019.

31. CCAP (Culture Collection of Algae and Protozoa). https://www.ccap. ac.uk/media/documents/BB.pdf. Accessed 20 Feb 2019.

32. Mastuti R, Rosyidah M. In vitro enzymatic isolation of protoplasts from tissues of the medicinal plant Physalis angulata L. AIP Conf Proc. 2019. https ://doi.org/10.1063/1.5061838.

33. Evans PK, Cocking EC. Isolation plant protoplasts. In: Street HE, editor. Plant tissue and cell culture. Berkeley: University of California Press; 1977. p. 103-35.

34. Gilbert EJ, Shohet S, Calligari PDS. Studies on the effect of protoplast density and genotype mixing on cell regeneration. Ann Appl Biol. 1995;126:379-93.

35. Kuhlenkamp R, Müller DG. Isolation and regeneration of protoplasts from healthy and virus-infected gametophytes of Ectocarpus siliculosus (Phaeophyceae). Bot Mar. 1994;37:525-30.

36. ThermoFisher Scientific. GeneArt ${ }^{\mathrm{TM}}$ Chlamydomonas protein expression vector for expression of recombinant proteins in Chlamydomonas reinhardtii. https://assets.thermofisher.com/TFS-Assets/LSG/manuals/MAN0O 
09793_GeneArt_Chlamydomonas_Exp_Vector_UG.pdf. Accessed 10 Mar 2019.

37. Harrison SJ, Mott EK, Parsley K, Aspinall S, Gray JC, Cottage A. A rapid and robust method of identifying transformed Arabidopsis thaliana seedlings following floral dip transformation. Plant Methods. 2006;2:19.

38. Logemann E, Birkenbihl RP, Ülker B, Somssich IE. An improved method for preparing Agrobacterium cells that simplifies the Arabidopsis transformation protocol. Plant Methods. 2006;2:16.

39. Neupert J, Karcher D, Bock R. Generation of Chlamydomonas strains that efficiently express nuclear transgenes. Plant J. 2009;57:1140-50.

40. Larkin PJ. Purification and viability determinations of plant protoplasts. Planta. 1976;128:213-6.

41. Sambrook J, Russell DW. Estimation of cell number by hemocytometry counting. CSH Protoc. 2006. https://doi.org/10.1101/pdb.prot4454.

42. Reddy CRK, Migita S, Fujita Y. Protoplasts isolation and regeneration of three species of U/va in axenic culture. Bot Mar. 1989;32:483-90.

43. Lin CS, Hsu CT, Yang LH, Lee LY, Fu JY, Cheng QW, et al. Application of protoplast technology to CRISPR/Cas9 mutagenesis: from single-cell mutation detection to mutant plant regeneration. Plant Biotechnol J. 2018:16:1295-310.

44. Yoo SD, Cho YH, Sheen J. Arabidopsis mesophyll protoplasts: a versatile cell system for transient gene expression analysis. Nat Protoc. 2007;2:1565-72.

45. Cocking EC, Evans PK. The isolation of protoplasts. In: Street HE, editor. Plant tissue and cell culture. Berkeley: University of California Press; 1977. p. 100-21.

46. Eriksson TR. Protoplast isolation and culture. In: Fowke LC, Constabel F, editors. Plant protoplast. Boca Raton: CRC Press; 1985. p. 1-20.

47. Gerken HG, Donohoe B, Knoshaug EP. Enzymatic cell wall degradation of Chlorella vulgaris and other microalgae for biofuels production. Planta. 2013:237:239-53.

48. Chawla HS. Introduction to plant biotechnology. 2nd ed. Enfield NH: Science Publishers; 2002

49. Coelho SM, Scornet D, Rousvoal S, Peters N, Dartevelle L, Peters AF, et al. Isolation and regeneration of protoplasts from Ectocarpus. CSH Protoc. 2012. https://doi.org/10.1101/pdb.prot067959.

50. Dodds JH, Roberts LW. Experiments in plant tissue culture. 2nd ed. Cambridge: Cambridge University Press; 1985.

51. Gandhi R, Khurana P. Regeneration from leaf protoplasts of Arabidopsis thaliana ecotype estland. Indian J Exp Biol. 2001;39:705-9.

52. Hanover JW, Keathley DE. Genetic manipulation of woody plants. 1st ed. New York: Plenum Press; 1988

53. Ohiwa T. Preparation and culture of Spirogyra and Zygnema protoplasts. Cell Struct Funct. 1977;2:249-55.

54. Vardi A, Spiegel-Roy P, Galun E. Citrus cell culture: isolation of protoplasts, plating densities, effects of mutagens and regeneration of embryos. Plant Sci Lett. 1975;4:231-6.
55. Agrawal SC. Zoospore formation in the green alga Stigeoclonium pascheri. J Algal Biomass Utln. 2012;3:18-9.

56. Singh RN. Reproduction in Draparnaldiopsis indica Bharadwaja. New Phytol. 1942;41:262-73.

57. Abe J, Hirano N, Komiya A, Kanda N, Fujiwara A, Hori S, et al. Preparation of knockdown transformants of unicellular charophycean alga, Closterium peracerosum-strigosum-littorale complex. Bio-protocol. 2016;6:e1813.

58. Abe J, Hori S, Tsuchikane Y, Kitao N, Kato M, Sekimoto H. Stable nuclear transformation of the Closterium peracerosum-strigosum-littorale complex. Plant Cell Physiol. 2011;52:1676-85.

59. Sørensen I, Fei Z, Andreas A, Willats WG, Domozych DS, Rose JK. Stable transformation and reverse genetic analysis of Penium margaritaceum: a platform for studies of charophyte green algae, the immediate ancestors of land plants. Plant J. 2014;77:339-51.

60. Kamisugi Y, Schlink K, Rensing SA, Schween G, von Stackelberg M, Cuming $A C$, et al. The mechanism of gene targeting in Physcomitrella patens: homologous recombination, concatenation and multiple integration. Nucleic Acids Res. 2006;34:6205-14.

61. Schaefer DG. A new moss genetics: targeted mutagenesis in Physcomitrella patens. Annu Rev Plant Biol. 2002;53:477-501.

62. Kim SI, Veena JH, Gelvin SB. Genome-wide analysis of Agrobacterium T-DNA integration sites in the Arabidopsis genome generated under nonselective conditions. Plant J. 2007;51:779-91.

63. Kudla J, Bock R. Lighting the way to protein-protein interactions: recommendations on best practices for bimolecular fluorescence complementation analyses. Plant Cell. 2016;28:1002-8.

64. Waadt R, Kudla J. In planta visualization of protein interactions using bimolecular fluorescence complementation (BiFC). CSH Protoc. 2008. https://doi.org/10.1101/pdb.prot4995.

65. Becker B. Snow ball earth and the split of Streptophyta and Chlorophyta. Trends Plant Sci. 2013:18:180-3.

66. Fang L, Leliaert F, Zhang Z, Penny D, Zhong B. Evolution of the Chlorophyta: insights from chloroplast phylogenomic analyses. J Syst Evol. 2017:55:322-32.

67. Fucikova K, Leliaert F, Cooper ED, Skaloud P, D'Hondt S, De Clerck O, et al. New phylogenetic hypotheses for the core Chlorophyta based on chloroplast sequence data. Front Ecol Evol. 2014;2:63.

68. Klebs G. Die Bedingungen der Fortpflanzung bei einigen Algen und Pilzen. 1st ed. Jena: G. Fischer; 1896.

\section{Publisher's Note}

Springer Nature remains neutral with regard to jurisdictional claims in published maps and institutional affiliations.
Ready to submit your research? Choose BMC and benefit from:

- fast, convenient online submission

- thorough peer review by experienced researchers in your field

- rapid publication on acceptance

- support for research data, including large and complex data types

- gold Open Access which fosters wider collaboration and increased citations

- maximum visibility for your research: over $100 \mathrm{M}$ website views per year

At BMC, research is always in progress.

Learn more biomedcentral.com/submissions 